\title{
Erratum: Electron-ion collision spectroscopy: Lithium-like xenon ions [Phys. Rev. A 91, 012710 (2015)]
}

\author{
D. Bernhardt, C. Brandau, Z. Harman, C. Kozhuharov, S. Böhm, F. Bosch, S. Fritzsche, J. Jacobi, S. Kieslich, H. Knopp, \\ F. Nolden, W. Shi, Z. Stachura, M. Steck, Th. Stöhlker, S. Schippers, and A. Müller
}

(Received 18 July 2016; published 8 August 2016)

DOI: 10.1103/PhysRevA.94.029903

In our publication, in Tables IV and V as well as in Fig. 10, the experimental resonance strengths $S$ and the theoretical resonance strengths $S_{T}$ were scaled according to $S^{\prime}=S p^{2} n^{3}$ and $S_{T}^{\prime}=S_{T} p_{T}^{2} n^{3}$, respectively, to account for the dominant expected proportionalities. In these equations, $p$ is the momentum in the electron-ion center-of-mass frame, and $n$ the principal quantum number of the shell into which the incident electron is captured. In the calculation of $p^{2}=\left(E_{\mathrm{c} . \mathrm{m} .} / c\right)^{2}+2 m_{0} E_{\mathrm{c} . \mathrm{m}}$. by mistake the mass of the ion, that is, $m_{0, i}=136 \times 931.494061 \times 10^{6} \mathrm{eV} / c^{2}$ was used instead of the mass of the electron $m_{0, e}=510.998928 \times 10^{3} \mathrm{eV} / c^{2}$. Thus, within the three digits given, all scaled resonance strengths $S^{\prime}$ and $S_{T}^{\prime}$ in Tables IV and $\mathrm{V}$ should be divided by a constant value of $2.48 \times 10^{5}$. Since Fig. 10 is a plot of the scaled resonance strengths, this correction also applies to Fig. 10.

Please note, that this correction only applies to the scaled resonance strengths $S^{\prime}$ and $S_{T}^{\prime}$ and not to dielectronic recombination rate coefficients or unscaled resonance strengths used elsewhere in the paper. In addition, none of the conclusions of our paper are influenced by this correction. 